\title{
2'-Hydroxycinnamaldehyde induces apoptosis through HSF1-mediated BAG3 expression
}

\author{
HAI-ANH NGUYEN and SOO-A KIM \\ Department of Biochemistry, Dongguk University College of Oriental Medicine, Gyeongju 780-714, Republic of Korea
}

Received September 27, 2016; Accepted November 29, 2016

DOI: 10.3892/ijo.2016.3790

\begin{abstract}
BAG3, a member of BAG co-chaperone family, is induced by stressful stimuli such as heat shock and heavy metals. Through interaction with various binding partners, BAG3 is thought to play a role in cellular adaptive responses against stressful conditions in normal and neoplastic cells. 2'-Hydroxycinnamaldehyde (HCA) is a natural derivative of cinnamaldehyde and has antitumor activity in various cancer cells. In the present study, for the first time, we identified that HCA induced BAG3 expression and BAG3-mediated apoptosis in cancer cells. The apoptotic cell death induced by HCA was demonstrated by caspase-7, -9 and PARP activation, and confirmed by Annexin V staining in both SW480 and SW620 colon cancer cells. Notably, both the mRNA and protein levels of BAG3 were largely induced by HCA in a dose- and timedependent manner. By showing transcription factor HSF1 activation, we demonstrated that HCA induces the expression of BAG3 through HSF1 activation. More importantly, knockdown of BAG3 expression using siRNA largely inhibited HCA-induced apoptosis, suggesting that BAG3 is actively involved in HCA-induced cancer cell death. Considering the importance of the stress response mechanism in cancer progression, our results strongly suggest that BAG3 could be a potential target for anticancer therapy.
\end{abstract}

\section{Introduction}

Various pathophysiological stresses can disrupt essential cellular signaling pathways and increase the concentration of unfolded or misfolded proteins. A cellular defense mechanism induces the expression of heat shock proteins (Hsps), which serve as molecular chaperones to refold the damaged proteins and protect native proteins $(1,2)$. The induction of stress-responsive genes is mainly regulated by heat shock transcription factor 1 (HSF1). Upon exposure to stressful

Correspondence to: Dr Soo-A Kim, Department of Biochemistry, Dongguk University College of Oriental Medicine, 707 Seokjangdong, Gyeongsangbuk-do, Gyeongju 780-714, Republic of Korea E-mail:ksooa@dongguk.ac.kr

Key words: BAG3, HSF1, apoptosis, stress, cinnamaldehyde, 2'-hydroxycinnamaldehyde stimuli, HSF1 translocates to the nucleus, where it binds to heat shock elements (HSEs) to induce the expression of Hsps $(3,4)$.

Bcl2-associated athanogene (BAG) 3 (also known as CAIR-1 or Bis) is a member of the BAG co-chaperone family. BAG family members share a conserved BAG domain in their C-terminal region, through which this family members interact with the ATPase domain of Hsp70 $(5,6)$. In addition to the BAG domain, BAG3 has a WW domain, two IPV motifs and a proline-rich (PXXP) motif. Through these motifs, BAG3 interacts with other binding partners such as Hsp22 and phospholipase $\mathrm{C}$ and participates in a wide variety of cellular processes including apoptosis, proliferation, migration and autophagy (6-11).

Among the six BAG family members, BAG3 is the only member induced by various cellular stresses such as oxidative stress, heat shock, heavy metals and HIV-1 infection (12-14). It has been reported that activated HSF1 induced the expression of BAG3 upon heat stress (15). Recently, we reported that BAG3 is a nuclear-cytoplasmic shuttling protein that interacts with HSF1 under both normal and heat-stressed conditions through its BAG domain. We also demonstrated that BAG3 affects HSF1 nuclear translocation and subsequent expression of target genes (16). HSF1 plays a central role in the cellular defense mechanism and Hsp70, a major stress-inducible protein, is elevated in many human cancers. Moreover, recent studies have shown that BAG3 levels are also elevated in various human cancers such as glioblastoma, leukemia, prostate and pancreatic cancer, suggesting the possibility that BAG3 may actively be involved in the initiation and progression of cancer (17-20).

Cinnamon, which is obtained from the stem bark of Cinnamomum cassia, has been widely used as flavoring and as a traditional medicine. Cinnamaldehyde is the major compound (45-65\% of the essential oil from bark) of cinnamon (21). As a major active compound, cinnamaldehyde has been well investigated and its diverse biological activities against bacteria, fungi, inflammation and tumor have been reported (22-25). 2'-Hydroxycinnamaldehyde (HCA), one of the natural derivatives of cinnamaldehyde, was reported to exert antitumor activity by inhibiting cell proliferation and inducing cell death in TNF- $\alpha$-treated colon cancer cells through the inactivation of NF- $\kappa$ B and AP-1 (26-28). Recently, we also demonstrated that HCA effectively induces apoptosis in human head and neck cancer cells in a p53-independent manner $(29,30)$. These 
previous reports suggest HCA as an effective therapeutic agent for treating cancer.

In the present study, we investigated the role of co-chaperone BAG3 on HCA-induced apoptosis in SW480 colon cancer cells and demonstrated the novel role of BAG3 on chemotherapy-induced cancer cell death.

\section{Materials and methods}

Cell culture and reagents. SW480 and SW620 human colon carcinoma cell lines were cultured in Dulbecco's modified Eagle's medium (DMEM; HyClone Laboratories, Inc., Logan, UT, USA) supplemented with $10 \%$ fetal bovine serum (FBS), 100 units/ml penicillin, and $100 \mu \mathrm{g} / \mathrm{ml}$ streptomycin (Gibco-BRL, Grand Island, NY, USA). Cells were maintained at $37^{\circ} \mathrm{C}$ in a humidified atmosphere with $5 \% \mathrm{CO}_{2}$. $\mathrm{HCA}$ was purchased from Santa Cruz Biotechnology (Santa Cruz, CA, USA) and dissolved in $0.1 \%$ dimethyl sulfoxide (DMSO).

Cell viability assay. The effect of HCA on cell viability was assessed using the trypan blue exclusion assay. SW480 and SW620 cells were seeded on a 12-well plate at a density of $3 \times 10^{5}$ cells $/ \mathrm{ml}$. The cells were cultured overnight and then treated with various concentrations of HCA. After $24 \mathrm{~h}$, the cells were trypsinized and stained with trypan blue dye (Sigma-Aldrich, St. Louis, MO, USA). The number of viable cells was counted using a hemocytometer.

Western blot analysis. SW480 and SW620 cells were treated with various concentrations of HCA for the indicated time periods. The cells were then washed with ice-cold phosphatebuffered saline (PBS) twice and lysed in RIPA buffer (PBS supplemented with $1 \%$ NP-40, $0.5 \%$ sodium deoxycholate, $1 \mathrm{mM}$ phenylmethylsulfonyl fluoride, $1 \mu \mathrm{g} / \mathrm{ml}$ aprotinin and $1 \mathrm{mM}$ sodium orthovanadate). Alternatively, the cytoplasmic and nuclear fractions were obtained according to the previously described method (16). The protein samples were resolved by SDS-polyacrylamide gel electrophoresis and then transferred onto nitrocellulose membranes. The membranes were blocked and then incubated with antibodies. Antibodies against PARP (sc-7150) and HSF1 (sc-9144) were purchased from Santa Cruz Biotechnology. The caspase-7 (\#9492), caspase-9 (\#9502), p38 (\#9212), ERK (\#4695), JNK (\#9258), phospho-p38 (\#9211), phospho-ERK (\#4370) and phospho-JNK (\#4668) antibodies were from Cell Signaling Technology (Danvers, MA, USA). Antibodies against BAG3 (ab47124) and TBP (ab818) were purchased from Abcam (Cambridge, UK), and antibodies against actin (A1978) were purchased from Sigma-Aldrich. Antibodies for BAG3 and actin were diluted 1:10,000 and all other antibodies were diluted 1:5,000. The immunoreactive bands were detected using the SuperSignal West Pico Chemiluminescent Substrate (Thermo Fisher Scientific, Rockford, IL, USA). All the experiments were performed at least 3 times.

RNA isolation and RT-PCR. SW480 cells were treated with $50 \mu \mathrm{M}$ HCA for the indicated time periods. Total RNA was isolated using TRIzol ${ }^{\circledR}$ reagent (Invitrogen, Carlsbad, CA, USA) according to the manufacturer's instructions. Semiquantitative RT-PCR was conducted using the One Step
RT-PCR PreMix kit (iNtRON Biotechnology, Seongnam, Korea) according to the manufacturer's instructions. The specific primers used for RT-PCR were as follows: BAG3, forward primer (5'-GAAAGTGGAAGCCATCCTGGA-3') and reverse primer (5'-CCCAAGTTACTGCATACCAAG CG-3'); GADPH, forward primer (5'-CCAAGGTCATCCAT GACAACTTTG-3') and reverse primer (5'-GTCATACCAG GAAATGAGCTTGACA-3'). RT-PCR was performed under the following conditions: 1 cycle of $30 \mathrm{~min}$ at $45^{\circ} \mathrm{C} ; 1$ cycle of $5 \mathrm{~min}$ at $94^{\circ} \mathrm{C}$; and 27 cycles of $30 \mathrm{sec}$ at $94^{\circ} \mathrm{C}, 30 \mathrm{sec}$ at $56^{\circ} \mathrm{C}$, and $40 \mathrm{sec}$ at $72^{\circ} \mathrm{C}$; with a final extension at $72^{\circ} \mathrm{C}$ for $5 \mathrm{~min}$. The PCR products were then electrophoresed on a $1.7 \%$ agarose gel and visualized by ethidium bromide staining.

siRNA experiment. SW480 cells were seeded in a 12-well plate at a density of $1.5 \times 10^{5}$ cells $/ \mathrm{ml}$ and then transfected with $1.5 \mu \mathrm{g} / \mathrm{ml}$ of either siGENOME BAG3 siRNA or HSF1 siRNA (Dharmacon, Lafayette, CO, USA) using the DharmaFECT transfection reagent (Dharmacon) according to the manufacturer's instructions. A siGENOME non-targeting siRNA pool (Dharmacon) was used as a control. After $24 \mathrm{~h}$, the cells were treated with $50 \mu \mathrm{M}$ HCA and incubated for an additional $24 \mathrm{~h}$. The cells were harvested, and the expression levels of BAG3 and HSF1 were analyzed by western blot analysis as described above.

Luciferase reporter gene assay. To assess the effects of HCA on HSF1 activity, SW480 cells were co-transfected with pGL3-Hsp70-Luc and pCH110 using FuGENE ${ }^{\circledR}$ HD (Promega, Madison, WI, USA) according to the manufacturer's instructions. After $8 \mathrm{~h}$, the cells were treated with various concentrations of HCA and incubated for an additional $24 \mathrm{~h}$. The cells were lysed with the reporter lysis buffer (Promega), and luciferase activity was measured using a Luciferase activity assay kit (Promega) according to the manufacturer's instructions. $\beta$-galactosidase activity was determined to normalize the luciferase activities.

Annexin V staining. SW480 cells were seeded on eight-chamber slides at a density of $1.5 \times 10^{5}$ cells $/ \mathrm{ml}$. Cells were cultured overnight and transfected with $1.5 \mu \mathrm{g} / \mathrm{ml}$ of either siGENOME BAG3 siRNA or a non-targeting siRNA pool as described above. Twelve hours after transfection, the cells were treated with $50 \mu \mathrm{M}$ HCA. After $24 \mathrm{~h}$, the cells were washed with PBS and stained using the Annexin V-Fluos staining kit (Roche Molecular Biochemicals, Indianapolis, IN, USA) according to the manufacturer's instructions. Cell nuclei were stained with DAPI (Molecular Probes, Eugene, OR, USA). The apoptotic cells were analyzed by conventional fluorescence microscopy (Axio Observer D1; Carl Zeiss, Oberkochen, Germany).

Statistical analysis. The data are expressed as the means \pm SD. ANOVA and the Student's t-test were applied to determine the statistical significance. $\mathrm{P}<0.01$ were considered to be significant.

\section{Results}

HCA induces the apoptotic cell death on SW480 and SW620 colon cancer cells. We first evaluated the effect of HCA on 
A

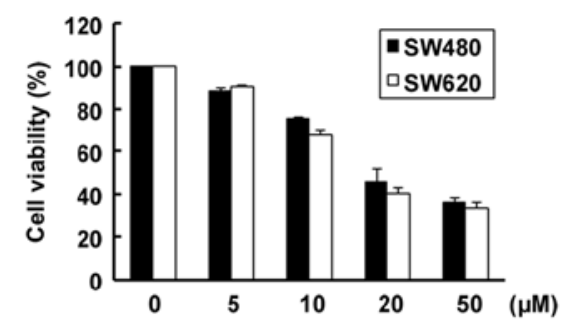

B

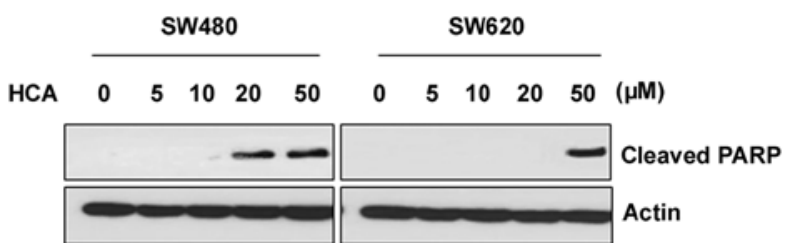

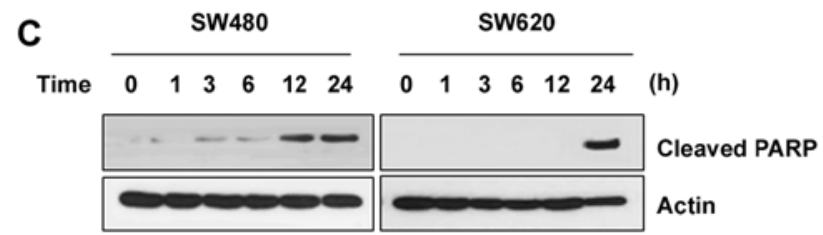

D
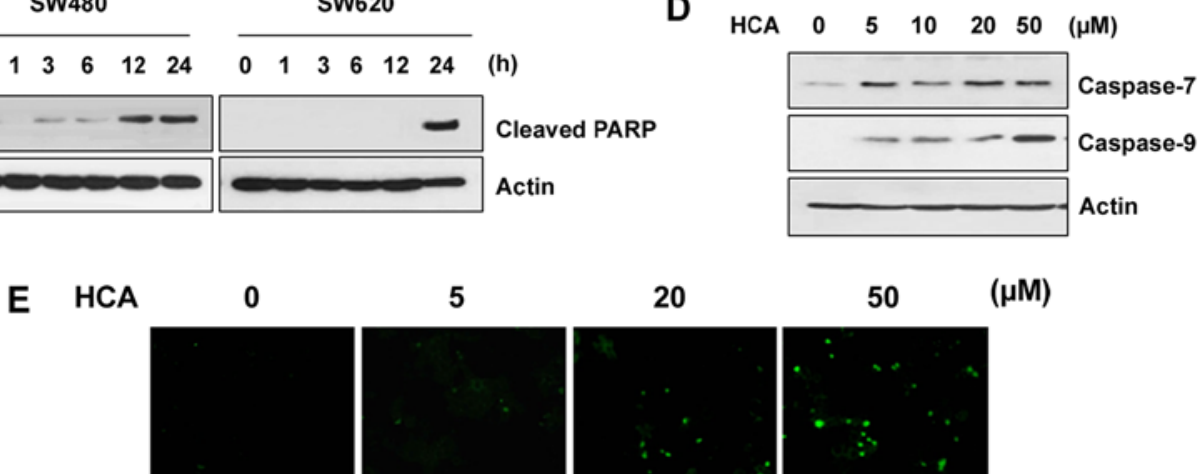

5

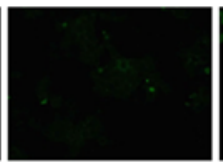

20

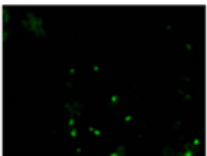

50

$(\mu \mathrm{M})$

Figure 1. HCA induces apoptosis on human colon cancer cells. (A) SW480 and SW620 cells were treated with various concentrations of HCA for $24 \mathrm{~h}$ and the cell viability was assessed by the trypan blue exclusion assay. The data are expressed as the means \pm SD of three individual experiments. (B and C) SW480 and SW620 cells were treated either with the indicated concentrations of HCA for $24 \mathrm{~h}$ (B) or with $50 \mu \mathrm{M}$ HCA for the indicated time periods (C). Total cell extracts were prepared, and the levels of cleaved PARP were detected by western blot analysis. (D) SW480 cells were treated with various concentrations of HCA for $24 \mathrm{~h}$. Total cell extracts were prepared, and the levels of caspase-7 and caspase- 9 were detected by western blot analysis. (E) SW480 cells were treated with the indicated concentrations of HCA for $24 \mathrm{~h}$ and stained with Annexin V-FITC. The cells were observed by fluorescence microscopy.

the viability of SW480 and SW620 colon cancer cells. The cells were treated with various concentrations of HCA for $24 \mathrm{~h}$ and the trypan blue exclusion assay was performed. As shown in Fig. 1A, cell viability was decreased depending on the HCA concentration in both SW480 and SW620 cells with $\mathrm{IC}_{50}$ values 18.7 and $16.5 \mu \mathrm{M}$, respectively. To address whether HCA induces apoptotic cell death in both cell lines, the cells were treated with HCA and examined for the levels of cleaved PARP. As shown in Fig. 1B and C, the levels of cleaved PARP were largely induced by HCA in a dose- and time-dependent manner. Consistent with the changes in the cleaved PARP levels, caspase-7 and caspase-9 were also increased depending on the HCA concentration (Fig. 1D). By staining SW480 cells with Annexin V-FITC, we confirm the role of HCA in caspase-mediated apoptosis (Fig. 1E).

$H C A$ induces the expression of BAG3. Previous studies have shown that the BAG3 expression can be induced by cellular insults such as heat shock, heavy metals and oxidative stress $(12,13)$. Therefore, we investigated whether HCA, a chemotherapeutic agent, can induce BAG3 expression. Notably, HCA largely increased the protein level of BAG3 in a dose- and time-dependent manner in both SW480 and SW620 cells (Fig. 2A and B). To investigate whether the increased protein levels of BAG3 are caused by increased BAG3 gene transcription, SW480 cells were treated with $50 \mu \mathrm{M}$ HCA for various times, and semi-quantitative RT-PCR was performed. As shown in Fig. 2C, HCA largely increased the level of BAG3 mRNA between 3 and $6 \mathrm{~h}$ of treatment. By showing that cycloheximide, an inhibitor of protein synthesis, effectively inhibited the HCA-induced levels of BAG3 protein expression, we confirmed that HCA promotes BAG3 gene expression (Fig. 2D).

HCA induces HSF1 transcriptional activity and subsequent BAG3 expression. Our new finding that HCA induces BAG3 expression leads us to investigate the signaling pathway involved in BAG3 expression. Previously, we and others have shown that the stress-responsive induction of BAG3 occurs mainly through the activation of $\operatorname{HSF} 1(15,16)$. To address the signaling mechanism responsible for HCA-induced BAG3 expression, we performed a reporter gene assay to evaluate the effect of HCA on the transcriptional activity of HSF1. SW480 cells were transfected with a pGL3-Hsp70-Luc luciferase reporter vector and then treated with various concentrations of HCA. Interestingly, HCA markedly promoted HSF1 transcriptional activity in a dose-dependent manner (Fig. 3A). As shown in Fig. 3B, HCA treatment resulted in rapid nuclear translocation of HSF1, suggesting that HCA effectively induced the transcriptional activity of HSF1. To further confirm the HSF1-mediated expression of BAG3 by HCA, the cells were transfected with HSF1-specific siRNA. As shown in Fig. 3C, the level of HSF1 was reduced by HSF1 siRNA. Consistent with the result shown in Fig. 2, HCA increased the level of BAG3. However, knockdown of HSF1 effectively inhibited the induction of BAG3 by HCA, confirming that HCA activates the transcription factor HSF1 and subsequent BAG3 expression.

Role of BAG3 on HCA-induced cell death. To investigate the role of BAG3 on the HCA-induced apoptotic cell death, SW480 cells were transfected with BAG3-specific siRNA. 


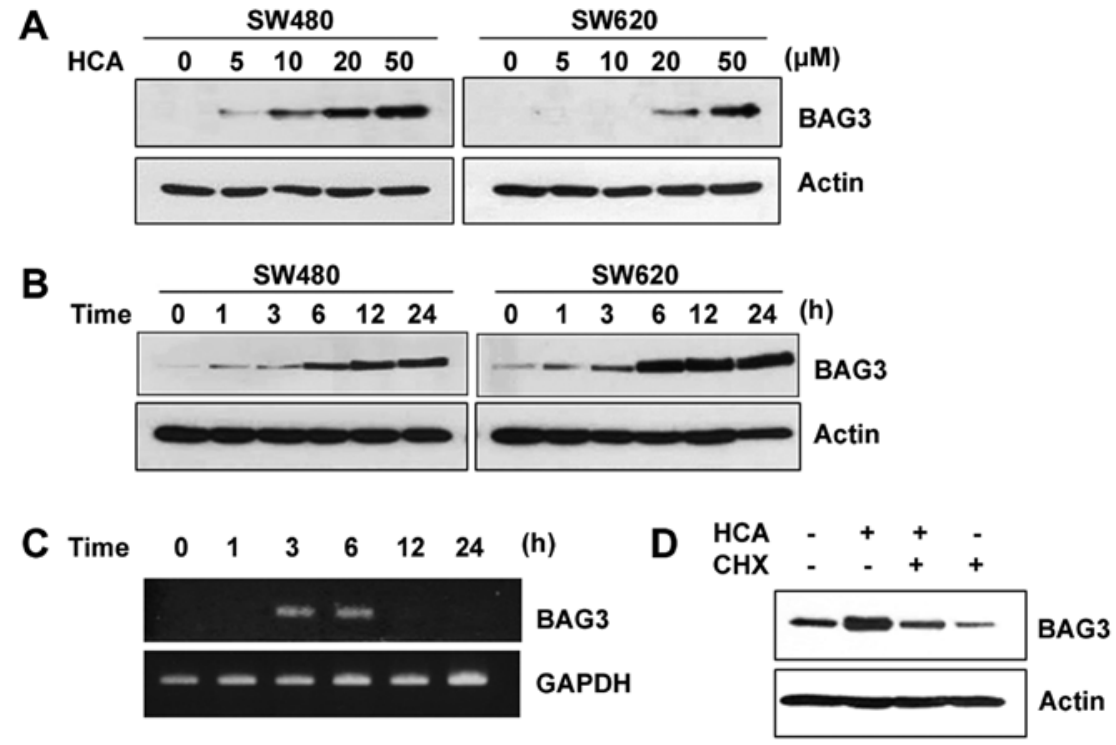

Figure 2. HCA induces the expression of BAG3. SW480 and SW620 cells were treated either with the indicated concentrations of HCA for $24 \mathrm{~h}$ (A) or with $50 \mu \mathrm{M}$ HCA for the indicated time periods (B). Total cell lysates were prepared and the expression level of BAG3 was detected by western blot analysis (C) SW480 cells were treated with $50 \mu \mathrm{M}$ HCA for the indicated time periods. Total RNA was isolated, and semi-quantitative RT-PCR was performed. GAPDH was used as an internal control. (D) SW480 cells were pretreated with cycloheximide for $4 \mathrm{~h}$ followed by treatment with $50 \mu \mathrm{M} \mathrm{HCA}$ for $24 \mathrm{~h}$. Total cell lysates were prepared and the levels of BAG3 were detected by western blot analysis. Actin was used as a loading control.

A

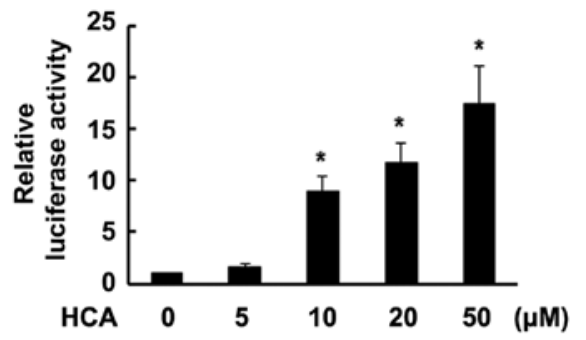

B
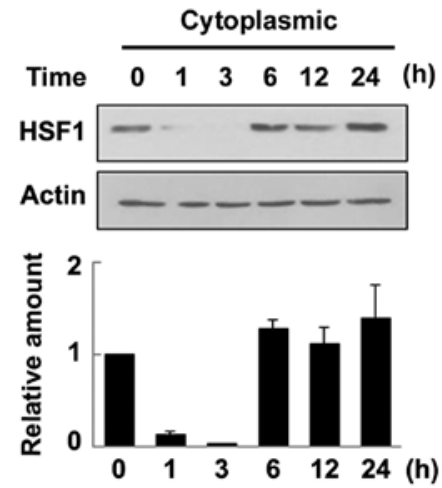

C

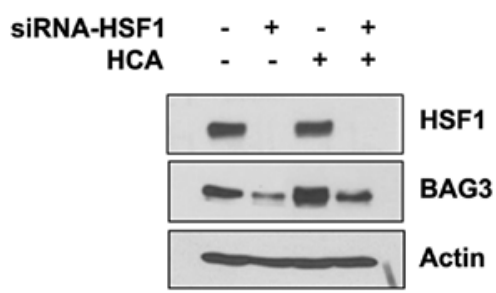

Nuclear
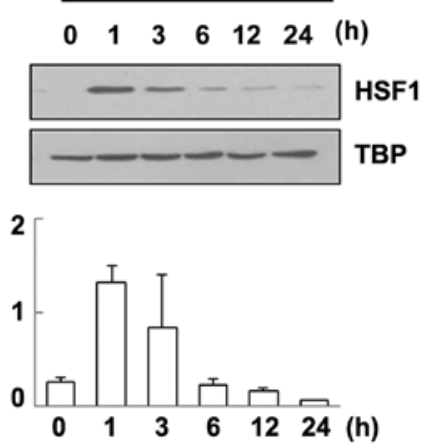

Figure 3. HCA promotes the activation of HSF1. (A) SW480 cells were transfected with a pGL3-Hsp70-Luc reporter vector. After 8 h, cells were treated with various concentrations of HCA for $24 \mathrm{~h}$. Total cell extracts were subjected to the luciferase assay. Luciferase activities were normalized for transfection efficiency by co-transfection with the $\beta$-galactosidase-expressing vector $\mathrm{pCH} 110$. The data were expressed as the means \pm SD of three individual experiments. ${ }^{*} \mathrm{P}<0.01$ compared with HCA-untreated control cells. (B) The cells were treated with $50 \mu \mathrm{M}$ HCA for the indicated time periods. Cytoplasmic and nuclear fractions were prepared, and the levels of HSF1 were detected by western blot analysis. The band densities were quantified by densitometry. The data are expressed as the means \pm SD of three individual experiments. Actin and TBP were used as loading controls. (C) The cells were transfected with the HSF1 siRNA constructs. After $24 \mathrm{~h}$, the cells were treated with $50 \mu \mathrm{M}$ HCA for $24 \mathrm{~h}$. The total cell lysates were prepared, and the levels of HSF1 and BAG3 were detected by western blot analysis.

As shown in Fig. 4A, HCA-induced BAG3 levels were dosedependently decreased by the BAG3 siRNA. Interestingly, the increased level of cleaved PARP by HCA was also decreased in BAG3 siRNA-transfected cells, suggesting that BAG3 is actively involved in the HCA-induced apoptosis (Fig. 4A). To further confirm this role of BAG3, cells were transfected with BAG3 siRNA and stained with Annexin V-FITC. As shown in Fig. 4B, HCA largely increased the number of apoptotic 
A

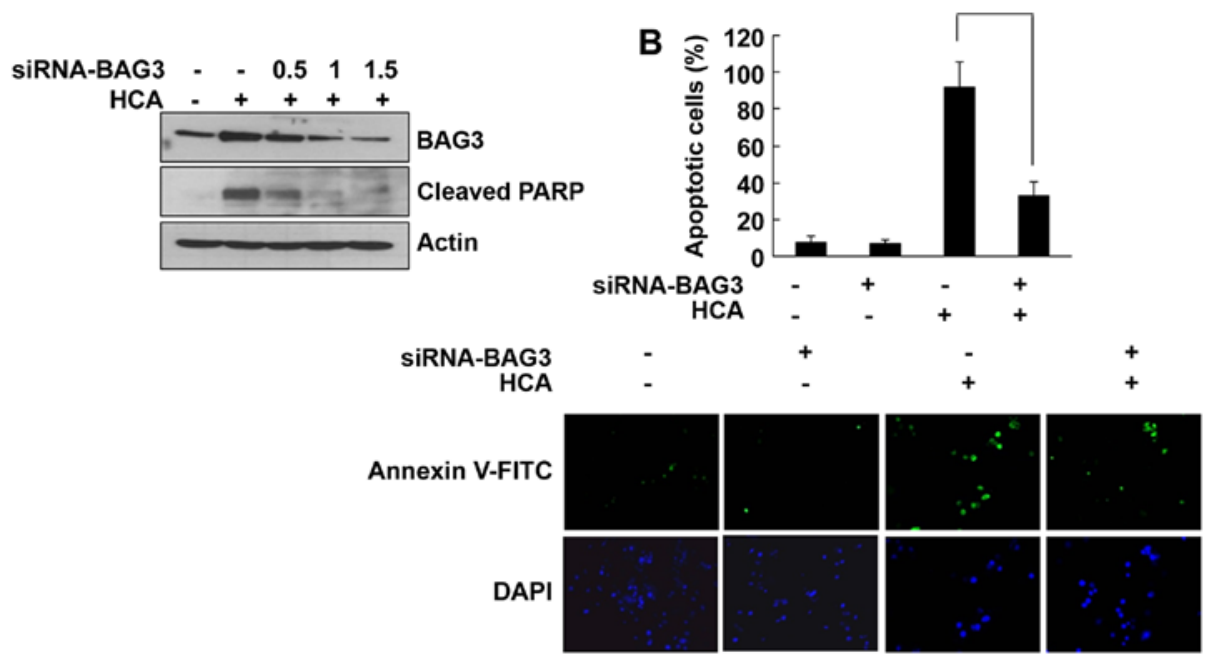

Figure 4. BAG3 is actively involved in HCA-induced apoptosis. SW480 cells were transfected with the indicated concentrations of BAG3 siRNA constructs. After $24 \mathrm{~h}$, the cells were treated with $50 \mu \mathrm{M}$ of HCA for $24 \mathrm{~h}$. (A) Total cell lysates were prepared and the levels of BAG3 and cleaved PARP were detected by western blot analysis. (B) The cells were stained with Annexin V-FITC and DAPI and observed by fluorescence microscopy. The data were expressed as the means \pm SD of three individual experiments. ${ }^{*}<0.01$ compared with HCA-treated cells.

A

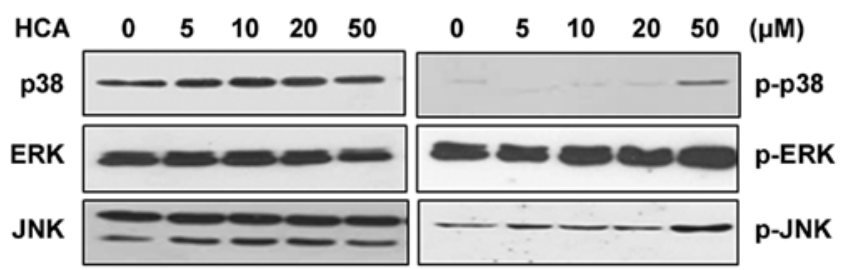

B

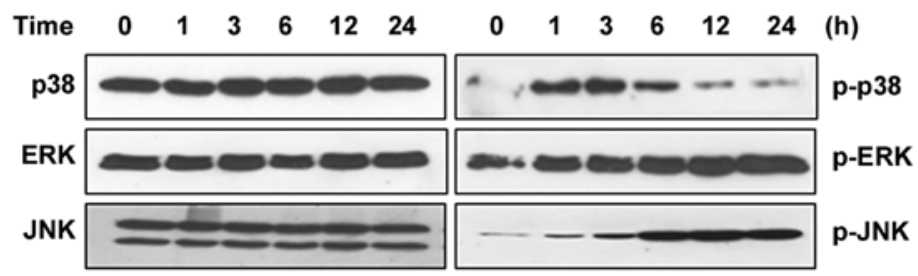

C
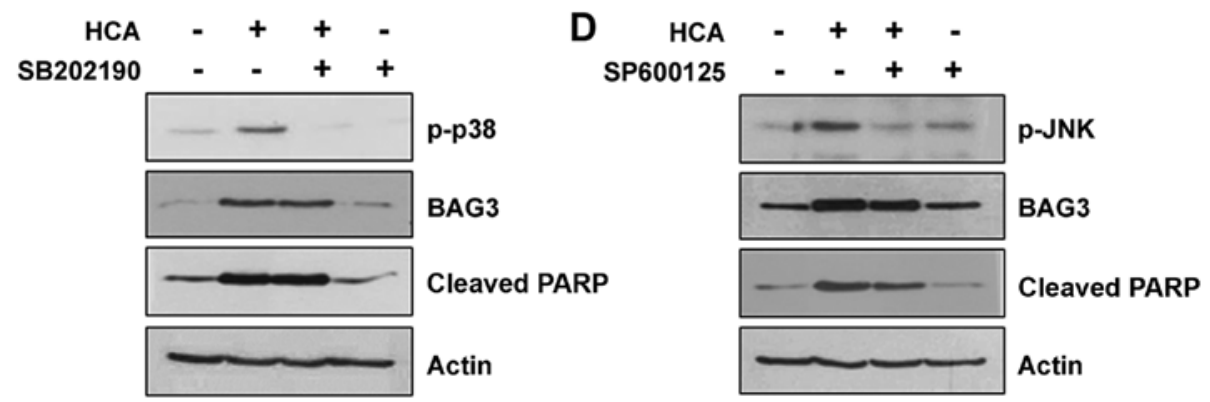

Figure 5. Effect of HCA on the MAPK signaling pathway. (A and B) SW480 cells were treated either with the indicated concentrations of HCA for $24 \mathrm{~h}$ (A) or with $50 \mu \mathrm{M}$ HCA for the indicated time periods (B). The levels of p38, ERK, JNK, p-p38, p-ERK and p-JNK were detected in total cell lysates by western blot analysis. (C and D) The cells were pretreated with either SB202190 for $2 \mathrm{~h}$ (C) or SP600125 for $1 \mathrm{~h}$ (D) followed by the addition of $50 \mu \mathrm{M}$ HCA for $24 \mathrm{~h}$. Total cell lysates were prepared and the levels of p-p38, p-JNK, BAG3 and cleaved PARP were detected by western blot analysis. Actin was used as a loading control.

cells. However, BAG3 knockdown effectively decreased apoptotic cells, suggesting that BAG3 is actively involved in HCA-induced apoptosis.

MAPK signaling pathways are not relevant to the induction of BAG3 expression. We next examined the MAPK signaling pathways in the HCA-treated SW480 cells to verify the involvement of MAPKs in HCA-induced BAG3 expression and/or apoptosis. HCA treatment had no detectable effect on the level of phosphorylated ERK. However, the levels of phosphorylated p38 and JNK were increased depending on the HCA concentration (Fig. 5A). Similarly, phosphorylated p38 and JNK were increased in a time-dependent manner, suggesting that p38 and/or JNK signaling pathways may be involved in 


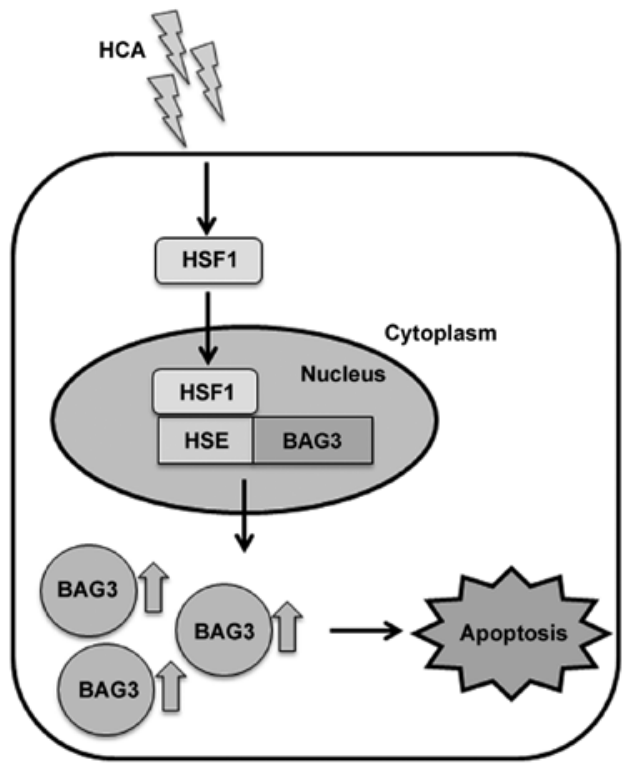

Figure 6. Schematic representation of the anticancer mechanisms of HCA in human colon cancer cells. HCA induces rapid nuclear translocation of HSF1 which lead to the expression of BAG3. Induced BAG3 is actively involved in caspase-dependent apoptotic cell death.

HCA-induced BAG3 expression and/or apoptosis (Fig. 5B). To confirm this possibility, cells were treated with either SB202190 or SP600125, which are specific inhibitors against p38 and JNK, respectively. As shown in Fig. 5C, SB202190 effectively inhibited phosphorylated $\mathrm{p} 38$; however, it did not affect HCA-induced BAG3 expression, suggesting that the p38 signaling pathway is not relevant to the HCA-induced BAG3 expression. Consistent with the level of BAG3, HCA-induced increases in cleaved PARP was not affected by SB202190, confirming that BAG3 is a major player in HCA-induced apoptosis. Similarly, although SP600125 effectively inhibited JNK phosphorylation, BAG3 induction was unaffected (Fig. 5D). The levels of HCA-induced cleaved PARP was also largely unaffected by SP600125, which supported the role of BAG3 on HCA-induced apoptosis.

\section{Discussion}

Heat shock response is one of the most evolutionary conserved cell protective mechanisms. Environmental insults induce various responses to help cells adapt to stressful conditions. HSF1 is placed in a center of the control of cellular responses to stress. In stressful conditions, HSF1 induces expression of Hsps, which are molecular chaperones that prevent protein aggregation and promote the refolding of misfolded proteins. If the cellular stress is too severe and misfolding exceeds a certain threshold, a signal that leads to apoptosis is activated thereby providing a balance between survival and death $(1-4,31)$.

Due to high levels of proteotoxic stress, the stress responsive pathway is important for cancer cell survival and proliferation. In this regard, it is not surprising that elevated levels of Hsps are commonly observed in a wide range of human tumors and that heat shock response is considered as a potential target for anticancer therapies $(31,32)$. However, the multifaceted outcomes of the HSF1-mediated stress response hinders the understanding of which stress-related signaling pathways are activated under certain circumstances, with regard to whether HSF1 plays a supportive or inhibitory role in cancer progression.

We demonstrated that HCA strongly induces apoptotic cell death in both SW480 and SW620 colon cancer cells. Interestingly, HCA largely increased both protein and mRNA levels of BAG3 in a dose- and time-dependent manner. This induction of BAG3 expression led us to investigate the involvement of the HSF1-mediated signaling pathway. Our data showed that HCA increased target gene promoter activity and nuclear translocation of HSF1. Furthermore, by showing that knockdown of HSF1 inhibited HCA-induced BAG3 expression, we verified that HCA strongly induced HSF1-mediated BAG3 expression. Considering the role of HSF1 in cellular protection and the elevated level of Hsp70 in many human cancers, our results suggest that BAG3 may be actively involved in cancer progression.

Importantly, BAG3 knockdown clearly verified that HCA promoted apoptosis via BAG3 expression. BAG3 is a stressinducible co-chaperone protein, and its expression level is elevated in several human cancers $(12,13,17-20)$. Previously, Liu and colleagues (33) have shown that apoptosis induced by bortezomib, a proteasome inhibitor, is greatly potentiated by BAG3 silencing in leukemic cells. Mani et al (34) also showed that BAG3 knockdown sensitized bladder cancer cells to apoptosis induced by ABT-737, a BH3 mimetic. These reports characterized BAG3 as an anti-apoptotic protein. However, contrary to the above, we showed that HCA induced apoptosis by increasing BAG3 expression.

Normally, HSF1-mediated cellular defense mechanisms protect the cells under stressful conditions. However, if the cellular stress is too severe to overcome, the cell undergoes apoptotic cell death. It is difficult to conclude whether the cellular defense mechanism plays a supportive or inhibitory role in cancer progression because this mechanism appears to have cell specificity. Even within the same cell type, cells can respond differently depending on its placed circumstances. BAG3 has many functional domains through which BAG3 interacts with other proteins (6-11). Our result showed that BAG3 critically participated in HCA-induced apoptosis and suggests the possible role of BAG3 as a key player in orchestrating the role of interacting protein(s) under given cell conditions.

In the present study, we verified that HCA induces apoptotic cell death in SW480 colon cancer cells. To the best of our knowledge, this is the first report describing that HCA induces apoptosis through the activation of HSF1 and subsequent BAG3 expression (Fig. 6). Further studies on BAG3 and its interacting proteins under stress conditions will reveal the role of BAG3 as a co-chaperone. Considering the importance of the stress defense mechanism on cancer progression, our results suggest that BAG3 is a potential target for development in cancer therapy.

\section{Acknowledgements}

The present study was supported by the Basic Science Research Program through the National Research Foundation 
of Korea (NRF) funded by the Ministry of Education, Science and Technology (2010-0023366).

\section{References}

1. Parsell DA and Lindquist S: The function of heat-shock proteins in stress tolerance: Degradation and reactivation of damaged proteins. Annu Rev Genet 27: 437-496, 1993.

2. Kaufman RJ: Molecular chaperones and the heat shock response. Sponsored by Cold Spring Harbor Laboratory, 6-10 May 1998. Biochim Biophys Acta 1423: R13-R27, 1999.

3. Morimoto RI: Regulation of the heat shock transcriptional response: Cross talk between a family of heat shock factors, molecular chaperones, and negative regulators. Genes Dev 12: 3788-3796, 1998.

4. Pirkkala L, Nykänen P and Sistonen L: Roles of the heat shock transcription factors in regulation of the heat shock response and beyond. FASEB J 15: 1118-1131, 2001

5. Kabbage $\mathrm{M}$ and Dickman MB: The BAG proteins: A ubiquitous family of chaperone regulators. Cell Mol Life Sci 65: 1390-1402, 2008

6. Rosati A, Graziano V, De Laurenzi V, Pascale M and Turco MC: BAG3: A multifaceted protein that regulates major cell pathways. Cell Death Dis 2: e141,2011.

7. Doong H, Price J, Kim YS, Gasbarre C, Probst J, Liotta LA, Blanchette J, Rizzo K and Kohn E: CAIR-1/BAG-3 forms an EGF-regulated ternary complex with phospholipase C-gamma and Hsp70/Hsc70. Oncogene 19: 4385-4395, 2000

8. McCollum AK, Casagrande G and Kohn EC: Caught in the middle: The role of Bag3 in disease. Biochem J 425: e1-e3, 2009.

9. Shi H, Xu H, Li Z, Zhen Y, Wang B, Huo S, Xiao R and Xu Z BAG3 regulates cell proliferation, migration, and invasion in human colorectal cancer. Tumour Biol 37: 5591-5597, 2016.

10. Suzuki M, Iwasaki M, Sugio A, Hishiya A, Tanaka R, Endo T, Takayama S and Saito T: BAG3 (BCL2-associated athanogene 3) interacts with MMP-2 to positively regulate invasion by ovarian carcinoma cells. Cancer Lett 303: 65-71, 2011.

11. Behl C: BAG3 and friends: Co-chaperones in selective autophagy during aging and disease. Autophagy 7: 795-798, 2011.

12. Pagliuca MG, Lerose R, Cigliano S and Leone A: Regulation by heavy metals and temperature of the human BAG-3 gene, a modulator of Hsp70 activity. FEBS Lett 541: 11-15, 2003.

13. Rosati A, Di Salle E, Luberto L, Quinto I, Scala G, Turco MC and Pascale M: Identification of a Btk-BAG3 complex induced by oxidative stress. Leukemia 23: 823-824, 2009.

14. Rosati A, Leone A, Del Valle L, Amini S, Khalili K and Turco MC: Evidence for BAG3 modulation of HIV-1 gene transcription. J Cell Physiol 210: 676-683, 2007.

15. Franceschelli S, Rosati A, Lerose R, De Nicola S, Turco MC and Pascale M: Bag3 gene expression is regulated by heat shock factor 1. J Cell Physiol 215: 575-577, 2008.

16. Jin YH, Ahn SG and Kim SA: BAG3 affects the nucleocytoplasmic shuttling of HSF1 upon heat stress. Biochem Biophys Res Commun 464: 561-567, 2015.

17. Festa M, Del Valle L, Khalili K, Franco R, Scognamiglio G, Graziano V, De Laurenzi V, Turco MC and Rosati A: BAG3 protein is overexpressed in human glioblastoma and is a potential target for therapy. Am J Pathol 178: 2504-2512, 2011.

18. Zhu H, Wu W, Fu Y, Shen W, Miao K, Hong M, Xu W, Young KH, Liu P and Li J: Overexpressed BAG3 is a potential therapeutic target in chronic lymphocytic leukemia. Ann Hematol 93: $425-435,2014$
19. Ammirante M, De Laurenzi V, Graziano V, Turco MC and Rosati A: BAG3 is required for IKK $\alpha$ nuclear translocation and emergence of castration resistant prostate cancer. Cell Death Dis 2: e139, 2011

20. Liao Q, Ozawa F, Friess H, Zimmermann A, Takayama S, Reed JC, Kleeff J and Büchler MW: The anti-apoptotic protein BAG-3 is overexpressed in pancreatic cancer and induced by heat stress in pancreatic cancer cell lines. FEBS Lett 503: 151-157, 2001.

21. Shen Y, Jia LN, Honma N, Hosono T, Ariga T and Seki T: Beneficial effects of cinnamon on the metabolic syndrome, inflammation, and pain, and mechanisms underlying these effects - a review. J Tradit Complement Med 2: 27-32, 2012.

22. Kwon JA, Yu CB and Park HD: Bacteriocidal effects and inhibition of cell separation of cinnamic aldehyde on Bacillus cereus. Lett Appl Microbiol 37: 61-65, 2003.

23. Cheng SS, Liu JY, Chang EH and Chang ST: Antifungal activity of cinnamaldehyde and eugenol congeners against wood-rot fungi. Bioresour Technol 99: 5145-5149, 2008.

24. Chao LK, Hua KF, Hsu HY, Cheng SS, Lin IF, Chen CJ, Chen ST and Chang ST: Cinnamaldehyde inhibits pro-inflammatory cytokines secretion from monocytes/macrophages through suppression of intracellular signaling. Food Chem Toxicol 46: 220-231, 2008

25. Jeong HW, Han DC, Son KH, Han MY, Lim JS, Ha JH, Lee CW, Kim HM, Kim HC and Kwon BM: Antitumor effect of the cinnamaldehyde derivative CB403 through the arrest of cell cycle progression in the $\mathrm{G} 2 / \mathrm{M}$ phase. Biochem Pharmacol 65: 1343-1350, 2003.

26. Lee SH, Lee CW, Lee JW, Choi MS, Son DJ, Chung YB, Lee CK, Oh KW, Moon DC, Kwon BM, et al: Induction of apoptotic cell death by 2'-hydroxycinnamaldehyde is involved with ERK-dependent inactivation of NF-kappaB in TNF- $\alpha$-treated SW620 colon cancer cells. Biochem Pharmacol 70: 1147-1157, 2005.

27. Hong SH, Kim J, Kim JM, Lee SY, Shin DS, Son KH, Han DC, Sung YK and Kwon BM: Apoptosis induction of 2'-hydroxycinnamaldehyde as a proteasome inhibitor is associated with ER stress and mitochondrial perturbation in cancer cells. Biochem Pharmacol 74: 557-565, 2007

28. Lee CW, Lee SH, Lee JW, Ban JO, Lee SY, Yoo HS, Jung JK, Moon DC, Oh KW and Hong JT: 2-hydroxycinnamaldehyde inhibits SW620 colon cancer cell growth through AP-1 inactivation. J Pharmacol Sci 104: 19-28, 2007.

29. Kim SA, Sung YK, Kwon BM, Yoon JH, Lee H, Ahn SG and Hong SH: 2'-Hydroxycinnamaldehyde shows antitumor activity against oral cancer in vitro and in vivo in a rat tumor model. Anticancer Res 30: 489-494, 2010.

30. Ahn SG, Jin YH, Yoon JH and Kim SA: The anticancer mechanism of 2'-hydroxycinnamaldehyde in human head and neck cancer cells. Int J Oncol 47: 1793-1800, 2015.

31. Dai C, Whitesell L, Rogers AB and Lindquist S: Heat shock factor 1 is a powerful multifaceted modifier of carcinogenesis. Cell 130: 1005-1018, 2007.

32. Sherman MY and Gabai VL: Hsp70 in cancer: Back to the future. Oncogene 34: 4153-4161, 2015.

33. Liu P, Xu B, Li J and Lu H: BAG3 gene silencing sensitizes leukemic cells to Bortezomib-induced apoptosis. FEBS Lett 583: 401-406, 2009.

34. Mani J, Antonietti P, Rakel S, Blaheta R, Bartsch G, Haferkamp A and Kögel D: Knockdown of BAG3 sensitizes bladder cancer cells to treatment with the BH3 mimetic ABT-737. World J Urol 34: 197-205, 2016. 